\title{
La mejora de la comprensión lectora: un análisis interactivo
}

\author{
JOSÉ ANTONIO LEÓN \\ Universidad Autónoma de Madrid \\ il \\ Resumen
}

El presente trabajo analiza, desde un enfoque interactivo, algunas caracteristicas del texto $y$ del lector. Desde esta perspectiva se revisan algunos de los modelos de intervención encaminados a facilitar en el sujeto el producto final de la comprensión lectora. Como es sabido, esta intervención puede introducirse tanto desde el mismo material escrito, a través de técnicas y recursos sobre planes de escritura que aplica el escritor, como desde las características del lector, como es el conocimiento previo y el uso de estrategias lectoras más eficaces en los sujetos. Desde esta última perspectiva incidimos especialmente en aquellos entrenamientos de estrategias encaminadas a potenciar un conocimiento y aplicación de la estructura organizativa de los textos.

Palabras clave: Comprensión de textos, Instrucción de textos, Estrategias de comprensión, Estructura del texto, Textos expositivos.

\section{Improvement of reading comprehension: an interactive analysis}

\section{Abstract}

This paper provides an assessment of the interactive approach between text and reader features. Within this framework several intervention models designed to facilitate the subject's attainment of reading comprehension are reviewed. As is usually known, intervention procedures may be based either on the written material itself, by means of the writer's own techniques and resources, or on the use and modification of more effective reading strategies on the subject's part. From this latter perspective, training in strategies intended to foster the knowledge and application of the text organizational structure is emphasized.

Keywords: Text comprehension, Instruction, Comprehension strategies, Text structure, Expository text.

Agradecimientos: Parte de la revisión realizada en este artículo, así como los artículos experimentales que se presentan a continuación han sido extraídos de la tesis doctoral del autor, tesis que fue dirigida por el profesor Mario Carretero.

Dirección del autor: Universidad Autónoma de Madrid, Facultad de Psicología. Departamento de Psicología Básica, Social y Metodología. Campus de Cantoblanco. 28049 Madrid. 1991.

Original recibido: Noviembre 1990. Revisión recibida: Junio 1991. Aceptado: Septiembre 


\section{INTRODUCCION}

Aunque el interés de la psicología por la lectura y la comprensión de textos no puede considerarse como algo nuevo, sí podemos afirmar que ha sido a partir de estas dos últimas décadas donde su estudio se ha abordado de una manera intensa y profunda. Durante todo este período y debido a su incesante aporte teórico y práctico, la psicología de la lectura y de la comprensión del material escrito ha estado sometida a una constante evolución.

La adopción de un enfoque constructivista de la memoria (véase León y García Madruga, 1991), la elaboración de herramientas analíticas con las que inspeccionar y diseccionar la estructura del material escrito, han sido, sin duda, factores que posibilitaron que este fenómeno se produjera. Sobre estos objetivos se diseñaron en los años setenta diversos modelos de análisis estructurales de la prosa, de los que sobresalen los análisis proposicionales propuestos para el estudio de textos narrativos y expositivos (Kintsch, 1974; Meyer, 1975; Kintsch y van Dijk, 1978) y los que se centraron en el estudio de historias populares y que se denominaron "gramáticas de las historias» (Rumelhart, 1975; Mandler y Johnson, 1977; Thorndyke, 1977; Stein y Glenn, 1979) (véase para un análisis más detallado, León, 1986).

Asimismo, la aceptación de conceptos y constructos que, como la teoría del esquema y de los modelos mentales, están permitiendo explicar de manera coherente cómo se representa y comprende el conocimiento en la mente del sujeto, cómo se almacena, así como de los procesos y estrategias que entran en juego. Una buena prueba de ello son los modelos explicativos e interactivos propuestos por algunos autores durante la década de los ochenta y que han considerado, además de la estructura del texto, los aspectos constructivos del lector (Johnson Laird, 1983; van Dijk y Kintsch, 1983; Meyer, 1984, 1985a y b; Garnham, 1987; Kintsch, 1988).

Como fruto de las investigaciones y trabajos teóricos de estos últimos años podemos asumir algunos rasgos característicos de la lectura y comprensión lectora. Así por ejemplo, cuando leemos un texto llevamos a cabo una tarea cognitiva enormemente compleja que implica diferentes procesos que actúan coordinadamente sobre la información escrita. Más específicamente y de acuerdo con Kleiman (1982), desde la lectura de un texto impreso hasta su comprensión final se necesita: a), un conocimiento general del mundo y de las acciones humanas; b), un conjunto de procesos perceptivos y cognitivos entre los que se incluyen procesamientos de discriminación perceptual, la intervención de la memoria a corto plazo (MCP), una codificación en orden serial, la localización y dirección de la atención, y un procesamiento inferencial; c), procesos de comprensión del lenguaje entre los que se incluyen la recuperación e integración del significado de la palabra, el análisis sintáctico de frases, la determinación de referencias anafóricas y diversos ánálisis de la estructura del discurso.

Para articular todas estas fases y subfases necesarias para obtener la comprensión de lo leído, nuestra mente no procede de una manera estrictamente secuencial, es decir, que procesa primero una unidad perceptual básica hasta llegar a la interpretación global del texto, sino que por el contrario, utiliza un proceso interactivo, a través del cual, el lector deriva información simultáneamente desde los distintos niveles, integrando información léxica, sintáctica, semántica, pragmática, esquemática e interpretativa (Adams, 1980; Perfetti y Roth, 1981; Rumelhart, 1977). Así, estos procesos actúan de forma paralela, activándose tan pronto 
como accede a ellos alguna información; es decir, el análisis semántico no espera a que finalice el análisis sintáctico, sino que una vez que han sido reconocidas determinadas palabras, trata de establecer las relaciones de significado dentro de la oración, lo que se verá confirmado por el análisis sintáctico a la vez que servirá como pista para el mismo (Rumelhart y otros, 1986; McClelland y otros, 1986).

Al igual que otros procesos cognitivos, la comprensión lectora está acotada por la propia capacidad limitada del sistema de procesamiento de la información humana. Sin embargo, esta limitación se compensa, en el caso del lector maduro, por la automatización de algunos de los procesos que actúan en los niveles más bajos, tales como las funciones de codificación o decodificación, permitiendo dirigir la atención del lector a procesos de comprensión de alto orden (Adams, 1980; Kleiman, 1982). Por esta razón, la lectura, con la práctica, se convierte en un proceso estratégico. El sujeto hábil lee con un objetivo determinado a la vez que controla de manera continua su propia comprensión (Bower, 1982; Brown, 1980; Brown, Armbruster y Baker, 1986). Los buenos lectores son capaces de seleccionar su atención hacia aquellos aspectos más relevantes del pasaje y en afinar progresivamente su interpretación del texto.

Todo ello parece redundar en la idea de que la lectura y su comprensión se conciben hoy como un proceso complejo e interactivo, a través del cual, el lector construye activamente una representación del significado poniendo en relación las ideas contenidas en el texto con sus conocimientos. La comprensión final del texto viene entonces producida por la conjunción de al menos dos factores. De un lado, las características del material escrito, expresadas no sólo en los diferentes niveles lingüísticos, sino también por su contenido y su estructura. Así, en este lugar podríamos situar todo lo que dependiese del texto y del escritor para optimizar su comunicación. Dentro de este apartado se introducirían aquellas técnicas que resultasen útiles para resaltar la estructura del texto, simplificar su contenido o mejorar su organización. Todo ello redundaría en una mejor activación de los conocimientos previos del lector, a la vez que permitiría la construcción de la macroestructura del mismo. Por otro, las características del lector, sus conocimientos y habilidades lectoras que utiliza para extraer la información del texto. Este apartado incluiria las estrategias que el lector aplica al enfrentarse a la lectura del pasaje.

Esta tendencia dominante de los modelos interactivos de la actividad lectora ha calado, en estos últimos años, no sólo en trabajos que se han interesado por el análisis de las estrategias utilizadas por los lectores, sino también por su enseñanza, por elaborar modelos de instrucción encaminados a mejorar su aplicación. Actualmente se considera que las dificultades para comprender lo leido se deben, al menos en parte, a que las estrategias de comprensión y de aprendizaje que poseen los sujetos son inapropiadas o inexistentes para realizar correctamente la tarea. Puede deberse incluso a que el lector no sea consciente de las demandas de la tarea. Las implicaciones en este cambio de orientación están cuestionando también el modo de enseñar. El enseñante, además de transmitir al alumno los contenidos propios de la asignatura que imparte, debe ser capaz de incitar al alumno a que trabaje con esos contenidos de manera activa, implicándole directamente en la tarea.

Esta doble perspectiva nos será útil en el desarrollo de este artículo en el que desarrollaremos brevemente cada uno de estos enfoques, aunque limitándonos en algunas de estas técnicas centradas en adolescentes y adultos y en materiales expositivos. 


\section{LA MEJORA DE LA COMPRENSION INDUCIDA DESDE EL TEXTO}

La comprensión y el recuerdo final de la información leída a menudo depende de cómo haya sido elaborado el material textual. Una excesiva utilización de términos técnicos y conceptos abstractos por el escritor, una estructuración poco clara de las ideas expresadas en el texto o la poca familiaridad del lector con el tema o el contenido del pasaje pueden ser factores determinantes que conviertan la lectura en una tarea excesivamente ardua y difícil, especialmente en lectores no avezados. Para paliar situaciones como éstas, el escritor dispone de determinados recursos que pueden facilitar la tarea del lector cuando trata de identificar la información relevante del material escrito. Los tipos de ayudas analizados han sido muchos y su estudio se ha centrado principalmente en examinar cómo la intromisión de estas ayudas repercuten en el aprendizaje y comprensión de la información escrita.

Una de las diferentes perspectivas que iniciaron este tipo de estudios procede de la psicología educativa que, ya desde los 60 , se mostraba interesada en la repercusión que sobre el aprendizaje del material escrito ejercía una gama de procedimientos instruccionales tales como los Organizadores Previos (OP), títulos, sumarios, preguntas y objetivos (Ausubel, 1968; Ausubel y cols., 1978). La concepción de Ausubel, por ejemplo, está basada en el desarrollo de una teoría sobre el aprendizaje significativo (Ausubel, 1963, 1968; Ausubel, Novak y Hanesian, 1978). Desde esta perspectiva, los OP son esencialmente una técnica que permite obtener al lector una dimensión general de las nuevas ideas del texto presentadas en un mayor nivel de abstracción y generalidad (véase, para una revisión más exhaustiva, García Madruga y Martín Cordero, 1987; Oakhill y Garnham, 1988). Otras técnicas utilizadas en la mejora de la comprensión del texto han sido los títulos, sumarios o resúmenes. En el caso de que el texto resulte ambiguo al lector, la presencia de los títulos se hace imprescindible para activar el esquema del sujeto. Respecto a su funcionamiento, los títulos y resúmenes parecen sólo compartir algunos procesos (ver Brooks y Dansereau, 1983). Los resúmenes, a diferencia de los OP, son menos abstractos y generales y aunque no proporcionan un marco tan amplio como los OP, pueden ayudar a mejorar una visión general del material que incluye algún punto de su estructura y argumentos principales. También facilitan la comprensión después de haber sido leído el pasaje.

Los OP propuestos por Ausubel han estimulado a otros investigadores a perseguir cuestiones similares, pero su eficacia en el aprendizaje y posterior recuerdo, ha sido objeto de una importante polémica durante los años setenta. El método ha sido criticado por no poseer explicitamente principios definitorios y generales acerca de qué son los OP y de cómo se construyen. Ello ha producido resultados inconsistentes en multitud de estudios, variando considerablemente la calidad de estos organizadores de un estudio a otro (Mayer, 1979; Oakhill y Garnham, 1988). Por otra parte, el método adolece de un escaso control experimental (Frase, 1975; Reder, 1985).

Otro tipo de ayudas son los objetivos y preguntas que, pertenecientes a la tradición conductista, han sido promovidas por autores como Rothkopf (1976). Rothkopf procura manipular estas variables en el texto con el objetivo de situar al sujeto en las condiciones más favorables para que emerjan en él aquellas conductas que facilitan su aprendizaje. También en este caso existe una polémica so- 
bre su eficacia, ya que, especialmente los objetivos, parecen producir una mejora en el aprendizaje intencional a costa del aprendizaje incidental, es decir, los sujetos parecen recordar mejor aquellos contenidos que son resaltados por los objetivos, pero disminuyen su recuerdo en los restantes conceptos. Los resultados experimentales sobre la utilización de objetivos parecen mostrar que su eficacia depende de la naturaleza de los contenidos y de los conocimientos y habilidades de los sujetos. Asimismo, los resultados parecen indicar que la presentación parcial de los objetivos, justo antes de los contenidos a los que se refieren, mejora los resultados si los comparamos con la presentación total de los objetivos antes del texto.

Otro grupo de investigaciones, proveniente de la psicolingüística y psicología cognitiva, se ha interesado por la manipulación de ayudas tales como la simplificación de la sintaxis y el léxico, el uso de conectivas o fórmulas retóricas, la organización de los contenidos y las señalizaciones, y cómo éstas repercuten positivamente en la memoria y comprensión del lector (Meyer, 1975; Schnotz, 1984). Este tipo de ayudas tiene una relevancia particular en el caso de los textos educativos que, por lo general, suelen ser de carácter expositivo. Como es sabido, este tipo de texto conlleva una gran cantidad de información nueva para el lector, hecho que va a dificultar al alumno reconocer con facilidad y en todas las ocasiones la información relevante. Una de las ayudas más investigadas han sido las denominadas "señalizaciones" (Meyer, 1975; van $\mathrm{Dij}, 1979$, 1980; van Dijk y Kintsch, 1983). Las señalizaciones se identifican con la intromisión de aquellas palabras $\mathbf{u}$ oraciones del texto que, aunque no añaden nueva información al contenido del mismo, resaltan las relaciones lógicas dominantes y las ideas que determinan la macroestructura del pasaje, permitiendo al lector identificar con mayor claridad los aspectos principales del mismo (Meyer, 1975; Loman y Mayer, 1983). Siguiendo a Meyer (1975), pueden establecerse al menos cuatro tipos de señalizaciones:

a) Aquellas que ofrecen una presentación previa del contenido resumido en una frase en la que se expresa la información clave que va a ser tratada de forma más amplia inmediatamente después, mediante frases tales como "Las causas principales del descubrimiento de América fueron...» o «las ideas centrales de este artículo son...» Oraciones como éstas ayudan al lector a detectar las ideas relevantes del pasaje.

b) Aquellas que, también bajo la forma de resumen o sumario, se ofrecen al final del texto a modo de conclusión.

c) Señalizaciones, llamadas ordinales o numerales por algunos autores (Lorch, 1985; Lorch y Chen, 1986), suelen utilizarse cuando se presenta un argumento que expresa diversos puntos de vista o diversas partes. En este caso, cada uno de ellos suele ir precedido por un número, letra o palabra «e.g. «en primer lugar...»o "por último...»).

d) Palabras que expresan la perspectiva del autor, tales como «Desafortunadamente...» 0 «Cabe resaltar...».

Las señalizaciones, introducidas bajo alguna de estas formas en el texto, ayudan al lector a identificar de manera más clara la estructura general del pasaje. De esta manera, las señalizaciones proporcionan un marco conceptual al sujeto para utilizar selectivamente la información relevante y organizarla dentro de una representación coherente. Los números o las palabras ordinales, por ejemplo, son marcadores explícitos de la organización textual, siguen la información contenida 
en el texto sobre los diversos aspectos o puntos de vista tratados. Al igual que ellos, todos ofrecen conjuntamente una panorámica más amplia y clara de la macroestructura del material escrito (Lorch y Chen, 1986). Este efecto selectivo de la información recordada ha sido puesto de manifiesto, tanto en el uso de señalizaciones aisladas, ya sean éstas numéricas (Lorch, 1985; Lorch y Chen, 1986), conectivas lógicas (Irwin y Pulver, 1984) o títulos (Wilhite, 1986), como en la combinación de varias, tales como frases previas, conectivas y otros recursos (Loman y Mayer, 1983: Mayer, Cook y Dyck, 1984; Meyer, 1984; Meyer, Brandt y Bluth, 1980; Meyer y Rice, 1982; León, 1989; León y Carretero, en prensa).

A pesar de estos resultados alentadores, aún no se conoce con exactitud el alcance de estas y otras ayudas. Las propias limitaciones en las que se desenvuelven los trabajos revisados no permiten ser contrastados entre sí, ya que en cada caso no siempre se han manipulado los mismos tipos de técnicas, materiales y sujetos. Por ello, aún sé necesita un cuerpo de investigaciones que especifiquen más claramente las características y usos de tales técnicas. Por otra parte, la investigación debe dar cuenta de los efectos que pudiesen producir cuando se aplican a materiales más amplios que los estudiados, como sería el caso de los libros de textos completos. Asimismo, resultaría interesante realizar análisis empíricos que determinasen si la experiencia de los lectorès al trabajar con pasajes señalizados, produce algún efecto de generalidad o transferencia en estrategias que puedan resultar efectivas cuando se le presentan textos no señalizados.

\subsection{De la estrecha relación entre el texto y las características del lector}

Lo visto hasta ahora nos da una idea de la importancia que tiene la presentación de ciertos recursos y técnicas que, presentes en el texto, facilitan la comprensión del mismo. Sin embargo, no debemos olvidar que estas ayudas inciden en el lector de manera diferente en función de sus conocimientos, así como de las estrategias lectoras que aplica.

Numerosas teorías contemporáneas del aprendizaje postulan que los lẹctores maduros se muestran estratégicamente activos cuando intentan aprender el contenido del texto. Según Winne $(1982,1983)$, estos lectores tienen en cuenta dos tipos de estímulos durante la lectura: el contenido que debe ser aprendido y las señalizaciones que el escritor o en su caso, el profesor o instructor, proporciona con el fin de activar en el lector un determinado proceso estratégico. Si el lector no se percata de la señalización o aún percatándose de ella, no activa el/los procesos cognitivos necesarios, probablemente no obtendrá una interpretación correcta del texto.

Estas diferencias observables entre los lectores han llevado a los estudiosos del tema a establecer distinciones generales en el uso de estrategias que los lectores aplican ante el estudio y la lectura del texto. Una propuesta hace mención a la distinción entre lectura «superficial» y "profunda» (Marton y Saljo, 1984; Entwistle y Waterson, 1988). Los lectores que activan superficialmente el texto, se limitan a ojearlo reteniendo algunos hechos aislados que no conciernen a la estructura global de la argumentación del mismo. Los sujetos del segundo grupo, en cambio, buscan la estructura subyacente de la información textual, la cuestionan, y relacionan con su conocimiento previo y experiencia.

A pesar de la evidente diversidad en el uso de estrategias y en el resultado lector, las características de los lectores no suele tenerse en cuenta cuando se elaboran los libros de texto, incluso en casos en los que se han introducido diferen- 
tes tipos de ayuda. No se cuida el hecho de que los lectores difieran ampliamente en sus razones para cursar unos determinados estudios, en su habilidad y motivación, en sus métodos o hábitos de estudio. En uno de sus trabajos, Schallert y cols. (Schallert, Alexander y Goetz, 1988) analizaron varios textos introductorios de las carreras de Psicología y Biología, en un intento de describir el tipo de ayudas de las que se servían los distintos autores para facilitar la asimilación de conceptos de dichos textos (ver tabla 1). Estos autores concluyeron que, a pesar de la presencia de estas ayudas en los textos, su presencia no garantizaba por sí sola un efecto positivo en la asimilación de la información por parte del lector. Ello requeriría, además, una implicación activa del lector en la tarea. Así, por ejemplo, el autor podría sugerir al lector la elaboración de un resumen de lo leído con el objeto de construir una representación mental sobre alguna porción relevante del texto. Sin embargo, la tónica general de los autores ha sido la de introducir ayudas en los textos sin que se demande necesariamente una actividad extra específica a los lectores. En el material revisado en éste y otros estudios (véase, por ejemplo, Goetz, Alexander y Schallert, 1987), las técnicas que demandan alguna actividad extra se reducen a cuestiones a las que el lector debe responder. Estas preguntas suelen encontrarse al final del capítulo o texto, siendo frecuentemente ignoradas por el lector. Por otra parte, la formulación de tales cuestiones no son todo lo deseable que se quisieran (Turner, 1989).

Una posible solución a este problema ha sido la propuesta por parte de algunos autores sobre la creación de textos "coherentes" (Armbruster y Anderson, 1985; Hartley, 1990). Así, por ejemplo, en su elaboración se tendría especialmente en cuenta la adecuación lingüística del texto al sector de la población al que iría dirigido. Se introducirían asimismo, cuestiones al encabezamiento y final del texto, ejemplos, problemas y experiencias próximas al lector en orden a seguir más fácilmente la exposición. Esta mejora en la calidad de los textos im-

TABLA I

Técnicas utilizadas por distintos autores de cinco textos introductorios de Psicología y cinco de Biologia

\begin{tabular}{|c|c|c|}
\hline \multicolumn{2}{|c|}{ Frecuencia de uso (en porcentaje) } & \multirow{2}{*}{ Técnicas aplicadas por los autores } \\
\hline $\begin{array}{l}\text { Textos } \\
\text { de Psicología }\end{array}$ & $\begin{array}{c}\text { Textos } \\
\text { de Biología }\end{array}$ & \\
\hline 45 & 29 & \multirow{6}{*}{$\begin{array}{l}\text { Indicios que dirigen la atención del lector (e.g.,. } \\
\text { objetivos, cuestiones, letra en negrita o itálica). } \\
\text { Señales orientadas hacia el contenido y su orga- } \\
\text { nización (e.g., títulos, resúmenes, esquemas...) } \\
\text { Ayudas encaminadas a facilitar la elaboración de } \\
\text { la información (e.g., ejemplos, paráfrasis, aplica- } \\
\text { ciones, comentarios marginales...). } \\
\text { Ayudas que complementan la comunicación tex- } \\
\text { to-lector (e.g., tablas, gráficos, fotografías....) } \\
\text { Señales que relacionan el contenido del texto con } \\
\text { una información familiar para el lector (e.g., ci- } \\
\text { tas a pie de página, alusiones, comparaciones co- } \\
\text { tidianas...). } \\
\text { Ayudas orientadas a motivar al lector (comenta- } \\
\text { rios irónicos o con notas de humor, ilustracio- } \\
\text { nes, fotografías...). }\end{array}$} \\
\hline 25 & 31 & \\
\hline 10 & 22 & \\
\hline 5 & 11 & \\
\hline 33 & 5 & \\
\hline 6 & 2 & \\
\hline
\end{tabular}


plicaría un cambio sustancial en la forma de concebir el material escrito que afectarían incluso a los llamados textos «electrónicos» (Britton y Glynn, 1989).

\section{LA MEJORA DE LA COMPRENSION INDUCIDA DESDE LAS ESTRATEGIAS DE LOS SUJETOS}

Otro aspecto fundamental para la obtención de una adecuada comprensión es el poseer, por parte del lector, estrategias a través de las cuales sepa organizar la nueva información extraída del texto y relacionarla con la que ya se posee. Estas estrategias difieren en aquellos sujetos que disponen de distinto nivel de habilidad lectora, resultando, en muchos casos, inadecuadas para poder construir un esquema organizacional donde representar eficazmente la estructura y el contenido del material leído.

Si un objetivo prioritario de este tipo de investigaciones es tratar de mejorar la comprensión de estos sujetos, se hace necesario conocer de antemano la capacidad que los sujetos poseen en la adquisición y uso de la información y cuestionarse si esa capacidad es susceptible de mejorarse con el entrenamiento de estrategias que permitan procesar la información de una manera más eficaz. La mayoria de los métodos utilizados en la mejora de la comprensión del material escrito se basan en la asunción de que los lectores menos capaces se beneficiarán de la enseñanza de aquellas estrategias que los lectores maduros utilizan de forma natural (Oakhill y Garnham, 1988).

Aunque no existe una clasificación general que incluya todos los modelos instruccionales, podemos distinguir, programas de carácter general y programas específicos. Ambos casos se desarrollan bajo una combinación de estrategias o programas de intervención complejos en los que se instruyen o enseñan múltiples estrategias. La razón de ello obedece a que la efectividad de centrarse en una estrategia de manera aislada, es cuando menos, dudosa, fundamentalmente porque el lector, en realidad, hace uso de diferentes estrategias combinadas (Mandl y Schnotz, 1987). Las diferencias entre ambos tipos de programas se establecen fundamentalmente en la extensión de los mismos. Los programas generales pretenden cubrir un amplio espectro, válidos para cualquier tipo de material escrito y se nutren de una combinación amplísima que incluye todo un arsenal de estrategias y recursos que se supone afectan, en alguna medida, al proceso de comprensión lectora. Un ejemplo de estos programas son el SPQ3 de Robinson (1970) o el programa de instrucción de estrategias de aprendizaje propuesto por Dansereau y cols. (véase León y García Madruga, 1989; García Madruga y León, en prensa). Por el contrario, los programas específicos se centran en un conjunto de estrategias hacia un fin concreto, como potenciar el conocimiento previo o modificar estrategias de supervisión.

Dentro de estos últimos hemos seleccionado dos tipos de intervención, programas de supervisión en estrategias metacognitivas (Mateos, a y b, en este mismo número) y en estrategias organizativas basadas en la estructuración del texto (León, b y c, también en este mismo número). En ambas se analizan las distintas procedencias teóricas de las que parte, incluyen la elaboración y evaluación de dos programas de intervención que serán expuestos en detalle en los artículos siguientes. Nos centraremos seguidamente en algunos estudios interesados en instruir en el conocimiento y aplicación de la estructura de textos. 


\subsection{El uso del conocimiento previo: Instrucción sobre la estructura y organización de los textos expositivos}

Como afirman Rumelhart y Norman (1978), cuando un sujeto adquiere una nueva competencia, los elementos de su conocimiento se incrementan interconectándose entre sí, accediendo a la memoria en forma de "paquetes» (chunks) de información organizada de una manera coherente. Como resultado de este desarrollo, los nuevos items de información se estructuran e integran con los conocimientos ya organizados en la memoria, distribuyéndose en unidades más amplias. La extraordinaria habilidad que posee un experto de un campo específico para recuperar adecuadamente la información de la memoria se basa, precisamente, en esa estructuración de la información almacenada (Ericson y Staszews$\mathrm{ki}, 1988)$. Estas estructuras de conocimiento altamente organizadas, que por algunos autores han sido denominadas «esquemas» (Bartlett, 1932; Rumelhart, 1980), se subordinan unas en otras, se modifican y se reelaboran, posibilitando un nuevo avance en el pensamiento. El experto, a diferencia del sujeto no considerado como tal, es capaz de crear formatos representacionales que correlacionan con su habilidad para resolver problemas (Glaser y Bassok, 1989).

La competencia del lector para resolver problemas tales como el decidir qué parte de la información leída es la importante, la teoría del esquema sugiere que el lector aplica su conocimiento acerca de la estructura del texto y del contenido esquemático a la hora de seleccionar esa información relevante (van Dijk y Kintsch, 1983). De hecho, esta habilidad supone un criterio que permite establecer diferencias importantes en el tipo de representación que obtienen los lectores «expertos» de los «novicios». Frente a un tipo de representación "coherente» del lector experto, caracterizada por un respeto a las reglas de formación y a la estructura de los textos, los lectores menos competentes, presentan un tipo de representación fragmentada y lineal denominada «tema más detalles" (Scardamalia y Bereiter, 1984).

Una parte importante de los trabajos que se han desarrollado para instruir a sujetos infantes, adolescentes y adultos en la adquisición y uso de la base de conocimientos, se han centrado casi con exclusividad en enseñar sobre el conocimiento y uso de la organización de textos expositivos. No faltan razones que apoyen este hecho. La propia presencia del libro de texto en los ambientes educativos, presencia que aumenta en relación directa con el nivel académico, es una de ellas. Pero no es la única. Algunas características de los textos y en especial su estructura, han sido puestas de manifiesto como variables determinantes en la comprensión final del mismo en un importante cuerpo de trabajos experimentales. El descubrimiento del llamado «Efecto de los niveles» según el cual, la información situada en los niveles más altos de la estructura jerárquica del texto es mejor recordada que la situada en los niveles más bajos, avala la importancia de la estructura textual (Cirilo y Foss, 1980; Mandler y Johnson, 1977; Meyer, 1977). La organización del texto se descubre también como una importante variable de la estructura del texto que puede facilitar o penalizar el producto lector (Kintsch y Yarbrough, 1982). Por otra parte, y partiendo de las características del sujeto, la competencia en el uso de la estructura del texto establece diferencias en la edad y habilidad de los lectores (Brown y Smiley, 1977; Poulson, Kintsch, Kintsch y Premach, 1979; Winogrand, 1983). Así, investigaciones recientes han demostrado que el conocimiento previo que posee el lector juega un papel crítico sobre lo que se aprende de un texto. Hartley (1988), por ejemplo, 
detectó correlaciones importantes entre el conocimiento previo que poseían los adultos y la cantidad de información que fueron capaces de recordar en textos que poseían una temática relacionada con tales conocimientos. Esta influencia del conocimiento también se ha puesto de manifiesto en materias más específicas, como es el caso de textos con un contenido relativo a ciencias físicas (Dee-Lucas y Larkin, 1986). Así, el lector que aplica la estructura del autor en la elaboración de sus respuestas, obtiene un mejor recuerdo (Taylor, 1980; Meyer, Brandt y Bluth, 1980). Incluso puede, a través de la aplicación de sus conocimientos sobre la estructura del pasaje, cubrir las deficiencias de los textos mal escritos (Taylor y Samuels, 1983). Todos estos tipos de conocimientos orientan la atención del sujeto hacia la información relevante, ayudando a la obtención de una correcta comprensión del texto.

Por el contrario, los lectores menos capaces suelen partir de un fondo de conocimientos más limitado y menos estructurado que los lectores más competentes. Consecuentemente, cabe pensar que ayudar a incrementar ese fondo de conocimientos y proporcionarle esquemas donde organizar mentalmente tal información, podria mejorar su capacidad de comprensión. Entrenar en el uso de los conocimientos previos afecta a algunos de los déficit constatados en nuestro análisis de las diferencias entre lectores capaces e inmaduros (León, 1989). Así, a la dificultad para contrastar la información del texto con la del propio lector (Owings y cols., 1980) y, fundamentalmente, a la necesidad de crear un contexto asimilativo donde integrar la información nueva (Mayer, 1985), aspiran tanto a modificar la concepción de la tarea como a promover medios o instrumentos apropiados para alcanzar este objetivo. Un aspecto especialmente interesante y que deseamos resaltar en esta revisión es el hecho de que ayudar a reconocer la organización textual como armazón para codificar la información del texto, puede ser, a su vez, un medio para construir un modelo mental que dé sentido a tal información (Mayer, Cook y Dyck, 1984).

\subsection{Entrenamiento en la adquisición de un «esquema estructural» previo}

Como algunos autores señalan, en términos de la teoría del esquema, el principal determinante de lo que una persona es capaz de adquirir de la lectura es la información que extrae de la tarea y la relación que establece con la información que ya se posee. Si el lector no posee el esquema relevante o no sabe cómo activarlo, será incapaz de comprender correctamente el material. A este respecto, Bridge (1987) sugiere que el investigador debe plantearse al menos dos cuestiones: ¿ cómo se le puede proporcionar un esquema adecuado al estudiante que no dispone del suficiente conocimiento previo necesario para comprender un pasaje?, y en caso de que lo posea, ¿de qué manera se le puede ayudar para que sea capaz de relacionar sus estructuras de conocimientos existentes con la nueva información?

Al igual que ocurre en otros campos en los que el sujeto requiere poseer esquemas mentales donde ubicar un conocimiento organizado y específico acerca de algo, un esquema sobre la estructura del texto implica en el lector estar en posesión de un conocimiento acerca de cómo los autores estructuran sus ideas en el pasaje (Ohlhausen y Roller, 1988). Desde una perspectiva investigadora, la especificad-generalidad de los esquemas estructurales en los lectores, a menudo depende de las cotas que haya establecido el experimentador y de la propia estructura del material escrito con la que se trabaja. 
Desde una perspectiva general, algunos autores han trabajado sobre la estructura textual tratando de analizar y explicitar la forma en que se organizan las ideas dentro del pasaje. Autores como van Dijk (1980), por ejemplo, sugiere que el discurso escrito podría ser organizado dentro de una serie de metacategorías válidas para cualquier tipo de texto: Introducción, problema, solución, expresando esta última las reacciones a los problemas planteados. La evaluación, donde se revisarían las soluciones ofrecidas y la enunciación de la parte o partes que no han sido resueltas y la conclusión, cuyo acometido sería el reunir la información, resumiéndola. Estas metacategorías, aunque nada desdeñables, resultan demasiado generales para ser consideradas específicas de la prosa expositiva, ya que bajo esta estructura podría incluirse cualquier otro tipo de material escrito.

Desde una óptica mucho más específica, se situaría la propuesta de Dansereau y cols. (Dansereau, 1978; Dansereau y otros, 1980; Holley y otros, 1979). Desde hace ya algún tiempo, estos investigadores desarrollaron una técnica basada en una "red de conceptos» (networking), en la que el texto era descompuesto en segmentos que representaban algunos tipos de conexión interna entre las ideas del pasaje, del que estos autores seleccionaron varias, tales como la analogía. Los estudiantes universitarios que fueron instruidos en la identificación de estas conexiones entre ideas, aprendieron a reconocer los diferentes tipos de conexiones, aplicando esta técnica en frases, pasajes y en el propio texto. El entrenamiento que sólo constó de cuatro sesiones (cinco horas y media en total), produjo una mejora general en el recuerdo de las principales ideas en el grupo que recibió el tratamiento. Estos resultados fueron replicados ampliando el entrenamiento a 24 horas, obteniendo los mismos resultados (Dansereau, 1983). Pero aún más interesante nos resulta la propuesta que Brooks y Dansereau (1983) presentaron en otro contexto. Sobre la base de la existencia de un «esquema estructural» análogo al esquema de la historia propuesto años antes por Kintsch (1977), pero esta vez aplicado sobre textos expositivos, construyeron, a partir del análisis de varios manuales científicos, lo que podríamos denominar como la superestructura (la forma convencionalizada) que aparecía en los textos científicos, a lo que denominaron DICEOX. En el uso de este esquema que contenía las categorías descripción, historia, consecuencia, pruebas, otras teorias e información abier$t a$, situándose en esta última aquella información no asimilable a las otras categorías, se instruyó a un grupo de estudiantes de "college" (18-21 años), mientras que otro grupo se utilizó como control. Se les sometió a una fase de entrenamiento que duró dos semanas e incluyó sólo seis horas de instrucción. El grupo control, a diferencia del experimental, participó en otra experiencia semejante en duración y número de sesiones, pero cuyo contenido era irrelevante para la comprensión de los textos. La medición del efecto del entrenamiento se llevó a cabo mediante el recuerdo libre de un texto expositivo, que no estaba organizado expresamente según las categorías del DICEOX. Los resultados indicaron una superioridad del grupo experimental sobre el grupo control en el recuerdo de las ideas importantes, no encontrándose diferencias significativas en el recuerdo de las ideas menos relevantes.

Otro tipo de procedimiento ha sido utilizado por Cook (1982). El entrenamiento diseñado por esta autora parece ayudar a los sujetos de «college» (nivel equivalente a los primeros cursos de carrera universitaria) a identificar la estructura de prosa que aparece en los libros de texto científicos de química, física y biología. Seleccionó cinco estructuras: Generalización, a través de la cual el pasaje explica, clarifica o amplía alguna idea; Enumeración, en el que se describe 
una lista de hechos secuencialmente ordenados; Secuencia, especifica una serie de sucesos o pasos de un proceso; Clasificación, en el que el texto especifica distintos materiales agrupados en categorías o clases y Comparación/Contraste, a través del cual se examina la relación entre dos o más ideas. Una vez comprobado en un estudio preliminar que estos alumnos podían ser enseñados a clasificar pasajes atendiendo a estas cinco categorías, el siguiente paso fue desarrollar un programa de entrenamiento de 10 horas en el que los estudiantes fueron instruidos en reconocer estas estructuras de prosa y el contenido relevante de los textos de química que utilizaban normalmente. Los sujetos entrenados en este procedimiento mostraron una ganancia significativa en el recuerdo de la información de alto nivel y en la resolución de problemas en contraste con las obtenidas en el grupo control, que no recibió tratamiento alguno. Estas mejoras se mantuvieron incluso en tareas en las que se trabajaba con un material expositivo diferente al habitual, como fueron los textos de física y biología. Resultados semejantes se han detectado en un estudio más reciente con alumnos de los primeros años de carrera de químicas con sólo ocho horas de entrenamiento (Cook y Mayer, 1988). Todos estos resultados indican que este tipo de entrenamiento se muestra eficaz en ayudar al lector a detectar y asimilar la estructura principal en pasajes científicos.

Todos estos trabajos presentan algunas limitaciones a sus posibles aplicaciones, puesto que sólo dan cuenta de un área de contenidos y temas muy limitados, como son los textos expositivos relativos a materias de ciencias. Además, se debe tener presente que estos tipos de estructuras no siempre aparecen de manera explícita en todos los textos $y$, por esta razón, requieren enseñarse previamente si se desea obtener algún éxito en su uso, hecho que no sucede en el esquema de los cuentos (León, 1986; León y Marchesi, 1987). A pesar de todo, el esquema estructural parece tener una existencia real. Por esa razón, suponemos que los lectores que están sometidos a la experiencia lectora continuada en este tipo de material escrito, lo descubren y utilizan.

\subsection{El plan estratégico de Meyer y cols.}

Las formas organizativas propuestas por Meyer, respecto al grado de generalidad de las dos anteriores, se sitúan en un nivel más intermedio. A diferencia del modelo de Brooks y Dansereau, esta autora no se ha limitado a un único tipo de texto expositivo específico, como los libros de textos científicos, sino que ha tratado los pasajes expositivos en un sentido más amplio, dentro del cual ha intentado reconstruir su semántica en términos formales. Como una parte de esa reconstrucción, incluye la explicitación del armaźón global del texto, expresado en un conjunto limitado de predicados retóricos o formas organizativas. Meyer (1985a y b) ha propuesto cinco formas de organización en estos textos que corresponden a cinco tipos de relaciones lógicas capaces de organizar la información del pasaje de acuerdo con su estructura jerárquica. Una de ellas es la relación retórica de causación o covarianza (antecedente/consecuente) e indica una relación causal entre distintos hechos. Así, en la frase «El descubrimiento de América fue posible gracias a un largo proceso de exploración que llevaron a cabo portugueses y castellanos por el océano Atlántico durante todo el siglo XV», "el proceso de exploración" (antecedente), está relacionado causalmente con "el descubrimiento de América» (consecuente), a través del predicado retórico «fue posible (produjo)». Este tipo de vínculos aparece frecuentemente en los textos científi- 
cos. Una relación de comparación expresa las diferencias o semejanzas entre dos temas. Consta de tres formatos: la comparación de analogía, la alternativa y la adversativa. La relación de colección señala cómo las ideas o sucesos están relacionados dentro de un grupo sobre la base de una cualidad común, tales como la secuencia de sucesos organizada temporal o espacialmente. La secuencia temporal es particularmente utilizada como estructura de alto nivel en textos de historia. La relación retórica respuesta incluye los formatos de la observación y la contestación, la pregunta y la respuesta, el problema y la solución. Un requerimiento de esta estructura es que debe existir al menos alguna conexión en el contenido entre las proposiciones interrelacionadas. Los artículos científicos y cuentos populares suelen estar escritos bajo esta estructura. Las relaciones de descripción ofrecen una mayor información temática al añadir una introducción o un mayor número de detalles, atributos o una explicación complementaria. El formato "quién», "qué», «dónde», «cuándo» y "por qué», muy utilizado en los informes periodísticos, se aproxima a la especificación previa de esta estructura de alto nivel. $\mathrm{El}$ «quién» y el «qué» se refiere al agente, acción, paciente e instrumento en la proposición principal del tema, mientras que el "dónde», "cuándo» y "por qué», se refieren a explicaciones o introducciones específicas que los diferentes tipos de descripciones expresan en el tema.

Estas relaciones retóricas han sido identificadas por la autora como «esquemas cognitivos» (Meyer, 1985a y b). Estos esquemas cognitivos o retóricos tienen, según la autora, un carácter más abstracto y general que el esquema narrativo o el situacional, $\mathrm{y}$ en ellos se incluye el conocimiento sobre cómo el texto ha sido organizado, obteniendo del mismo una idea global. Por ejemplo, si el lector ante la presencia de un pasaje expositivo activase el esquema problema/solución, introduciría en él aquel contenido que se adecuase a las variables relativas del problema, tales como su descripción, antecedentes y consecuencias, y en la solución, aquella información relativa a las alternativas que bloquean al menos una de las causas del problema. Además, para recuperar la información una vez retenida, el lector activaría el mismo esquema superordinado utilizado anteriormente (Meyer, 1984, 1985a, 1987). Esta construcción opera de un modo estratégico en el lector maduro. El sujeto aprovecha las claves que posee el texto, permitiendo reconocer su patrón organizativo, la "relación retórica dominante" y, que una vez reconocida, se serviría de ella como armazón para ordenar y estructurar las proposiciones leídas en el texto. Esta estrategia, denominada «estrategia estructuralm, actuaría de una manera analítica, pues debido a la variabilidad o a la posibilidad de errar en la identificación de la estructura del texto, el lector confirmaría las hipótesis a partir de evidencias parciales o las rechazaría a medida que la información siguiente no se ajustara a ese armazón previamente construido. La estrategia estructural que suele instruirse incluye el reconocimiento de la estructura del texto expositivo, identifica las ideas relevantes del pasaje sobre el paso anterior. El conocimiento de la estructura del texto puede asistir a los lectores en la construcción de una representación mental de la información, describiendo en varios procesos de la comprensión lectora: selección de la información relevante del pasaje, procesamiento del conocimiento relevante existente, construcción de las conexiones internas entre los elementos seleccionados del pasaje y elaboración de conexiones externas entre lo seleccionado del pasaje y el conocimiento existente en la memoria del sujeto (Cook y Mayer, 1988).

Sobre esta base teórica, Meyer junto con sus colaboradores, han propuesto un modelo de instrucción, basado en lo que han denominado "plan estratégico" 


\section{8}

(Meyer y cols., 1989). A través de este plan, los lectores aprenden a emplear deliberadamente un grupo de estrategias dirigidas a recordar lo que ellos leen. Durante la lectura, el lector debe seleccionar el plan organizacional utilizado por el escritor y sobre éste debe organizar sus ideas. Cuando trata de recordar la información leída, el lector puede utilizar como forma de organizar su recuerdo el mismo plan organizacional desarrollado por el texto.

El primer programa de entrenamiento sistemático utilizado en sujetos adolescentes fue elaborado por Bartlett (1978). Esta autora diseñó este programa para enseñar a sujetos de noveno grado (nivel equivalente al de $10^{\circ}$ de BUP) la identificación y uso de cuatro estructuras expositivas, descripción, problema-solución, causación y comparación. La instrucción constaba de cinco sesiones de una hora. Cada sesión comenzaba con la lectura de un pasaje y una vez obtenido un esbozo general del mismo, el profesor suscitaba la discusión con los alumnos sobre la idea principal del mismo, utilizando como criterio las estructuras anteriormente mencionada (grupo experimental), o la discusión se centraba sobre aspectos del contenido relativamente poco importantes, sin apoyo en la estructura del texto (grupo control). Ambos grupos contaron con los mismos materiales y profesor. Los estudiantes del grupo experimental demostraron que con el uso de la estrategia se producía una mejora en su capacidad de memoria. Obtuvieron una superioridad respecto a sus niveles pre-test y sobre el grupo control. Estas diferencias en el uso de la estrategia y las mejoras en el recuerdo se mantuvieron en una prueba de recuerdo demorado de una semana.

Ha habido importantes estudios que han demostrado la validez de éste y otros métodos que han utilizado como base de su instrucción la organización de los textos. Una forma ha sido la de generar representaciones en forma de diagrama sobre las ideas básicas y sus relaciones del texto, como los diagramas de flujo (Geva, 1983), la aplicación en niños de modelos similares al propuesto por Meyer (Armbruster y cols., 1987) o con técnicas tales como el resumen jerárquico (Taylor, 1980). Geva (1983), por ejemplo, proporciona a sujetos de nivel universitario (18-21 años) un entrenamiento amplio ( 20 horas en 5 semanas) en la investigación de formatos de causación. Los sujetos utilizaron un procedimiento de diagramas de flujo para diseñar las ideas del texto o relacionarlas con su conocimiento previo, como nodos que permitieran especificar las relaciones entre ellas. Los resultados obtenidos en el post-test demostraron que los sujetos mejoraron significativamente su representación del contenido y la estructura del texto. Superaron a los sujetos del grupo control que recibieron entrenamiento en habilidad lectora, en la búsqueda de palabras claves y conjunciones. Barnet (1984), por su parte, ofreció a sujetos de este mismo nivel académico una breve descripción de una estructura del texto antes de leer un informe científico para facilitar así su localización en la memoria. Los sujetos recordaron significativamente más información en una prueba de recuerdo con demora de dos días que aquellos sujetos que recibieron la descripción de la estructura del texto después de haber leído el artículo científico, o sobre aquellos que no recibieron tal descripción.

La enseñanza de la estructura del texto también se ha aplicado en niños, como son los realizados por Armbruster, Anderson y Ostertag (1987). Estos autores han utilizado un procedimiento similar al plan estratégico de Meyer para enseñar a niños de quinto grado (nivel equivalente a 5. de EGB) sobre cómo utilizar la estructura problema-solución cuando trabajan con materiales de Ciencias Sociales. El modelo, al igual que el de Meyer, enseña la estructura del texto siguiendo los principios de instrucción directa, instrucción que incluye un modelado por 
el profesor sobre los procedimientos definidos explícitamente y una práctica orientada a incrementar y ampliar la dificultad de los textos, incluye técnicas correctoras de retroalimentación, obteniendo un éxito moderado.

Vidal-Albarca (1990) ha desarrollado un modelo de instrucción en alumnos de 5. ${ }^{\circ}$ curso de EGB basado en la captación de ideas principales en textos expositivos con una estructura enumerativa y comparativa, así como en la elaboración de la macroestructura. Con este entrenamiento, apoyado de una metodología de instrucción directa, los sujetos experimentales se mostraron superiores frente al grupo control en la habilidad de captar la idea explícita e implícita de los textos expositivos aunque no en todas las variables medidas. Esta mejora se mantuvo tras un período de cuatro meses. Resultados similares han sido obtenidos en otros estudios recientes (Sánchez, 1988, 1990). En este último, el programa de instrucción dirigido a alumnos de $6 .^{\circ}, 7 .^{\circ}$ y $8 .^{\circ}$ de EGB se centró en potenciar actividades cognitivas dirigidas a captar la progresión temática del texto, extraer el significado global a través de la aplicación de las macrorreglas de selección, generalización e integración (véase Kintsch y van Dijk, 1978; van Dijk y Kintsch, 1983) y a reconocer y aplicar la estructura principal del texto.

Otro procedimiento en niños ha sido el utilizado por Taylor (1980) y que ha denominado resumen jerárquico, que consiste en desarrollar tareas que preparan una estructura muy esquemática del texto. Taylor comparó su programa con un diseño tradicional de instrucción en comprensión de textos. Se inducía al niño a analizar la estructura gráfica del texto, representando gráficamente el esqueleto de la estructura de párrafo que eran listados con números romanos en el encabezamiento y apoyándose sobre este esquema, el alumno debía rellenar con las ideas principales cada una de las entradas. Posteriormente, Taylor y Beach (1984) trabajaron con este procedimiento en sujetos de nivel equivalente a $7 .^{\circ}$ de EGB recibieron instrucción y práctica en estas tareas durante siete horas, a razón de una hora por semana que aplicaron sobre materiales de Ciencias Sociales. Entre las tareas ralizadas, se incluía la de elaborar la idea general esquemática de toda la lectura como la de colocar títulos a las diferentes partes del texto. Los resultados indicaron que los estudiantes podrían realizar de esta manera resúmenes más significativos cuando eran capaces de localizar las ideas importantes del mismo.

Aunque resulta difícil establecer qué factores son los responsables de la mejora en la comprensión final de aquellos sujetos que han sido instruidos a través de una combinación de estrategias orientadas a inducir un conocimiento sobre la estructura y organización de los textos, la evaluación global parece resultar positiva. Así en general, podemos señalar que el poseer un conocimiento previo sobre la organización de los textos, mejora la comprensión de pasajes que al menos se adecuan a esa estructura. Desde el texto y a través de ayudas como las señalizaciones, pueden facilitar el desarrollo de este aspecto. Con estos procedimientos los estudiantes pueden estimular su capacidad para relacionar sus conocimientos con la nueva información del texto, permitiendo activar sus niveles más altos de procesamiento con la información textual.

\section{CONSIDERACIONES FINALES}

No ha sido nuestro objetivo ofrecer una revisión exhaustiva sobre las técnicas y programas encaminados a la mejora del aprendizaje y retención de textos, pero 
esperamos haber confirmado la idea de cómo la comprensión puede ser mejorada por este tipo de ayudas y procedimientos de entrenamiento que se muestran efectivos en lectores adolescentes y adultos. A pesar de ello, conviene tener presente algunas limitaciones propias de este tipo de trabajos.

Una primera restricción hace referencia a la generalidad de la ayuda o intervención aplicada. Sobre este respecto no consideramos que exista un «tipo de ayuda o estrategia ideal» universal y válida para todos los casos. Los factores del texto, del lector o de la situación contextual en la que se desarrolla el acto de leer pueden alterar el efecto de una estrategia. Así, por ejemplo, la edad y nivel de habilidad de un estudiante tiende a interactuar diferencialmente con el tipo de instrucción utilizada o la implantación de algunas de estas técnicas en niños, sólo producen un éxito moderado. Una limitación de las estrategias diagramas o cualquier otra representación gráfica, ha sido señalada por Armbruster y cols., (1987), en el sentido de que éstas no ayudan a identificar la estructura de alto nivel o macroestructural aunque el lector extraiga una estructura, ésta no tiene por qué contener lo esencial o la idea principal del texto. En este sentido, otros autores (Brown, Palincsar y Armbruster, 1984; Scardamalia y Bereiter, 1984; Carretero y León, 1990) han puesto de manifiesto que el dominio del procesamiento de los textos por los niños, no es algo que se adquiere rápida y fácilmente, y que es a partir de la adolescencia, cuando los sujetos pueden convertirse en lectores expertos capaces de extraer en profundidad la información que los textos contienen. Otra consideración relevante dentro del campo aplicado, algunos métodos son más practicables que otros. Además, conviene a veces dedicar más tiempo en la enseñanza de estrategias generales que introducirse en ayudas tales como gráficos o subtítulos cuando existen multitud de textos que no las poseen.

Otra limitación relacionada con la anterior se identifica con los esquemas estructurales. La mayoría de los textos expositivos no poseen algoritmos tan claros como, por ejemplo, los estudiados en las gramáticas de la historia. Consecuentemente, las expectativas acerca de lo que un pasaje expositivo contiene o no, no están tan claramente definidas como en un cuento popular. Las relaciones que se incluyen en los textos expositivos frecuentemente dependen de un orden lógico, que puede describir paradigmas retóricos de clasificación, comparación o contraste..., y éstas no se adecuan a un único formato universal. A pesar de ello y como hemos visto, algunos tipos de esquemas pueden resultarnos útiles en la interpretación correcta del material escrito, especialmente cuando el lector es capaz de elaborar o activar un esquema que se empareje con el establecido por el escritor en el pasaje. Este punto de vista nos ofrece algunas implicaciones en la instrucción, en el sentido que puede resultarnos factible orientar la instrucción en la reconstrucción, por parte del lector, de los esquemas del escritor.

$\mathrm{La}$ intervención en estrategias de comprensión basadas en el conocimiento y uso de la estructura textual puede ayudar notablemente a la comprensión del marco organizacional del autor y facilitar su aprendizaje. Puede asimismo, facilitar la elaboración de esquemas a modo de resolución de problemas, que resulten útiles al lector a la hora de cumplir sus objetivos de lectura en aquellas tareas que requieran la utilización de la información textual. Creemos que este tipo de técnicas pueden resultar muy útiles dentro del aula, hasta ahora más interesada en la adquisición de conocimiento previo y menos preocupada en adiestrar al alumno en estrategias o actividades que puedan facilitarle la comprensión de un texto escrito. 


\section{Referencias}

ADAMS, M. J. (1980). «Failures to comprehed and levels of processing in reading». En R. J. Spiro, B. C. Bruce y W. F. Brewer (ed.), Theoretical issues in reading comprehension. Hillsdale, Nueva Jersey: Erlbaum.

ARMBRUSTER, B. B. y ANDERSON, T. H. (1985). "Producing "considerate” expository text: Or easy reading is damned hard writing». Joumal of Curriculum Studies, 17 3, 247-274.

ARMBRUSTER, B. B., ANDERSON, T. H. y OSTERTAG, J. (1987). *Does text structure/summarization instruction facilitate learning from expository text? Reading Research Quarterly, 22, 331-346.

Ausubel, D. P. (1963). The Psychology of Meaningful Verbal Learning. Nueva York, Grune and Stratton.

- (1968). Educational Psycbology: A cognitive View. Nueva York: Holt, Riniehart y Winston.

Ausubel, D. P., NOVAK, J. D. y HANESIAN, H. (1978). Educational Psychology, 2.2 Ed., Nueva York: Holt Rinehart y Winston (Trad. cast. de M. Sandoval: Psicología Educativa. México: Trillas, 1983).

BARNETT, J. E. (1984). «Facilitating retention through instruction about text structure». Joumal of Reading Behavior, 16, 1-13.

BARTLETT, B. J. (1978). Top-level structure as an organizational strategy for recall of classroom text. Tesis doctoral no publicada. Arizona State University.

BARTLETT, F. C. (1932). Remembering. A study experimental and social psychology. Cambridge. Cambridge University Press.

BOWER, J. A. (1982). * Memory limitations in the oral reading comprehension of fourth-grade children*. Journal of Experimental Child Psychology, 34, 200-216.

BRIDGE, C. A. (1987). «Strategies for Promoting Reader-Text Interactions». En R. J. Tierney, P. L. Anders y J. N. Mitchell (eds.): Understanding Readers' Understanding. Hillsdale, Nueva Jersey: Erlbaum.

BRITTON, B. K. y GLYNN, S. M. (1989). Computer writing environments: Theory reserach and application. Hillsdale, N.J.: Erlbaum.

BroOKS, L. W. y DANSEREAU, D. F. (1983). «Effects of structural schema training and text Organization on Expository Prose Processing*. Joumal of Educational Psychology, 75, 6, 811-820.

BROWN, A. L. (1980). «Metacognitive development and reading». En R. J. Spiro, B. Bruce y W. Brewer (ed.): Theoretbical issues in reading comprehensión: Perspectives from cognitive psychology, linguistics, artificial intelligence and education. Hillsdale, Nueva Jersey; Erlbaum.

BROWN, A. L., ARMBRUSTER, B. B. y BAKER, L. (1986). *The role of metacognition in reading and studying». En J. Orasanu (ed.): Reading comprebension: from research to practice. Hillsdale, Nueva Jersey: Erlbaum.

BROWN, A. L. y SMILEY, S. S. (1977). *Rating the importance of structural units of prose passages: A problem of metacognitive development». Child Development, 48, 1-8.

CARRETERO, M. y LeÓN, J. A. (1990). «Desarrollo cognitivo y aprendizaje en la adolescencia». En J. Palacios (comp.), Psicologia Evolutiva, Madrid: Alianza.

CIRILO, R. y FOSS, D. (1980). *Text structure and reading time for sentences». Journal of Verbal Learming and Verbal Behavior, 19, 96-109.

CoOK, L. K. (1982). The effects of text structure on the comprebension of scientific prose. Tesis doctoral no publicada. University of California, Santa Bárbara.

COOK, L. K. y MAYER, R. (1988). *Teaching Readers about the structure of scientific textm. Journal of Educational Psychology, 80, 4, 448-456.

DANSEREAU, D. F. (1978). «The Development of a learning strategy curriculum». En H. F. O'Neil, Jr. (ed.): Leaming strategies. Nueva York: Academic Press, 1978.

- (1983). Leaming strategy research. (Technical Report). For Worth: Texas Christian University.

Dansereau, D. F., LONG, G. L., Evans, S. H. y ACTKINSON, T. R. (1980). *Objective ordering of instructional material using multidimensional scaling». Joumal of Structural Leaming, 6, 299-314.

DEE-LUCAS, D. y LARKIN, J. H. (1986), «Novice strategies for comprehending scientific texts». Discourse Processes, 9, 329-354.

ENTWISTLE, N. J. y WATERSON, S. (1988). «Approaches to studyng and levels of processing in university students*. British Journal of Educational Psychology, 58, 3, 258-265.

ERICSON, K. A. y STASZEWSKI, J. (1989). «Skilled memory and expertise: mechanisms of exceptional performancew. En D. Klahr y K. Kostovsky (eds.): Complex Information Processing: The impact of Herbert A. Simon. Hillsdale, N.J.: Erlbaum.

FRASE, L. T. (1975). *Advances in research and theory in instructional technology». En F. N. Kerlinger (ed.: Review of Research in Education, 3, Peacok.

Garcia Madruga, J. A. y León, J. A. (en prensa). «Comprensión y Memoria de Textos Expositivosw. En M. Carretero y J. A. García Madruga (comps.): Adolescencia y Aprendizaje. Barcelona: LAIA.

Garcla Madruga, J. y Martin CoRdero, J. I. (1987). Aprendizaje, comprensión y retención de textos. Madrid: UNED.

GARNHAM, A. (1987). Mental Models as representation of discourse and text. Chichester: John Wiley \& Sons. 
GEVA, E. (1983). «Facilitating reading through flowcharting». Reading Research Quarterly, 18, 384-405.

GLASER, R. y BASSOK, M. (1989). «Learning theory and the study of instruction*. Annual Review Psychology, 40, 631-666.

Glover, J. A., Dinnel, D. L., Halpain, D. R., McKee, T. K., Corkill, A. J. y Wise, S. L. (1988). «Effects of Across-Chapter Signals on Recall of Text*. Joumal of Educational Psychology, 80, 1, 3-15.

Goetz, E. T., Alexander, P. A. y Schallert, D. L. (1987). «The author's role in cueing strategic processing of college textbooksm. Reading Research and Instruction, 27, 1, 1-11.

HARTLEY, J. (1990). *Textbook design: Current status and future directions». International Journal of Educational Research, 14, 6, 533-541.

Holley, C. D., Dansereau, D. F., McDonald, B. A., Garland, J. C. y Collins, K. W. (1979). "Evaluation of a hierarchical mapping technique as an aid to prose processing*. Contemporary Educational Psychology, 4, 227-237.

IRWIN, J. W. y PULVER, C. J. (1984). «Effects of explicitness, clause order, and reversibility on children's comprehension of causal relationship". Joumal of Educational Psychology, 76, 399-407.

Johnson-LaIRD, P. N. (1983). Mental Models. Cambridge: Cambridge University Press.

KINTSCH, W. (1974). The representatiion of meaning in memory. Hillsdale, Nueva Jersey; Erlbaum.

- (1977). «On comprehending stories». En M. A. Just y P. A. Carpenter: Cognitive processes in comprehensión. Hillsdale, Nueva Jersey; Erlbaum.

- (1988). *The role of Knowledge in Discourse Comprehension: A Construction-Integration Modelm. Psychological Review, 95, 2, 163-182.

KINTSCH, W. y VAN DIJK, T. A. (1978). «Toward a model of text comprehension and production». Psychological review, 85, 363-394.

KINTSCH, W. y YARBROUGH, J. C. (1982). "The role of rhetorical structure in text comprehension*. Journal of Educational Psychology, 74, 828-834.

KLEIMAN, G. M. (1982). "Comparing good and poor readers: A critique of research". En K. E. Nelson (ed.): Children's language. Vol. 3. Hillsdale, Nueva Jersey: Eribaum.

LEÓN, J. A. (1986). La memoria de los niños a través de los chentos: un análisis experimental. UNED: Mérida.

- (1989). Comprensión y memoria de textos expositivos: Diferencias entre sujetos expertos y novatos. Tesis doctoral inédita. Madrid: Universidad Autónoma de Madrid.

LEON, J. A. y CARRETERo, M. (en prensa). «Signals effects on the recall and understanding of expository texts in expert and novice readers $»$. En A. J. M. Oliveira (ed.): Structures of comunication and intelligent belps for Hypermedia Couserware. Nueva York: Springer-Verlag.

LEÓN, J. A. y GARCIA MADRUGA, J. A. (1989). «Comprensión de Textos e Instrucción*. Cuadernos de Pedagogia, 169, 54-59.

- (1991). *Comprensión y Memoria de Textos». En J. M. Ruiz Vargas (comp.): La Psicología de la memoria. Madrid: Alianza Psicología.

LEÓN, J. A. y MARCHESI, A. (1987). «La influencia de variables cognitivas en el recuerdo de cuentos y su valoración en función de la edad*. Infancia y Aprendizaje, 37, 19-31.

LOMAN, N. C. y MAYER, R. (1983). *Signaling Techniques that increase the understability of expository prose». Joumal of Educational Psychology, 75, 402-412.

LORCH, R. F. JR. (1985). «Effects of recall of signals to text organization". Bulletin of the Psychonomic Society, 23, 374-376.

LORCH, R. F. JR. y CHEN, A. M. (1986). *Effects of Number Signals of Reading and Recall». Joumal of Educational Psychology, 78, 4, 263-270.

MANDL, H. y SCHNOTZ, W. (1987). «New directions in text comprehensionm. En E. De Corte, H. Lodavijks, P. Parmentier y P. Span (ed.): Learming and instruction: european research in an international context. Vol. 1 . Oxford: Pergamon.

MANDLER, J. M. y JOHNSON, N. S. (1977). «Remembrance of things parsed: Story structure and recallw. Cognitive Psychology, 9, 111-151.

MARTON, F. y SALjO, R. (1984). «Approaches to learning». En F. Marton, D. Hounsell y N. Entwistle (eds.): The experience of learning. Edinburgh: Scottish Academic Press.

MAYER, R.E. (1979). «Can advance organizers influence meaningful learning?» Review of Educational Research, 49, 371-383.

- (1985). «Structural analysis of Science prose: can we increase problem-solving performance». En B. K. Britton y J. B. Black: Understanding expository text. Hillsdale, Nueva Jersey: Erlbaum.

MAYER, R. E., COOK, L. K. y DYCK, J. L. (1984). «Techniques that help readers build mental models from scientific text: Definitions pretraining and signaling». Journal of Educational Psychology, 76, 1089-1105.

MCClelland, J. L., Rumelhart, D. E. y grupo PDP (1986). Parallel distributed processing. Explorations in the microstructure of cognition. Vol. 2. Cambridge, Ma: Bradford Books.

MEYER, B. J. F. (1975). The organization of prose and its effects on memory. Amsterdam: North-Holland.

- (1977). «What Is Remembered from Prose: A Function of Passage Structure». En R. O. Freedle (ed.): Discourse Processes: Advances in Research and Theory. Vol. 1: Discourse Production and Comprehension. Nueva Jersey: Ablex Publishing Corporation. 
- (1984). *Text dimensions and cognitive processing*. En H. Mandl, N. L. stein, y T. Trabasso (eds.): Learning and comprebension of text. Hillsdale, N.J.: Erlbaum.

- (1985a). *Prose analysis: Purposes, procedures and problems». En B. K. Britton y J. B. Black (eds.): Understanding Expository Text. Hillsdale, Nueva Jersey: Erlbaum.

- (1985b). *Prose analysis: Purposes, procedures and problems (Part II)». En B. K. Britton y J. B. Black (eds.): Understanding Expository Text. Hillsdale, Nueva Jersey: Erlbaum.

- (1987). «Following the author's top-level organization; and important skill for reading comprehension*. En R. J. tierney, P. L. Anders y J. N. Mitchell (eds.): Understanding reader's understanding: Theory and Practice. Hillsdale, Nueva Jersey: Erlbaum.

MEYER, B.J. F., BRANDT, D. M. y BLUTH, G. J. (1980). «Use of top-level structure in text: Key for reading comprehensión of ninth-grade students». Reading Research Quarterly, 16, 72-102.

MEYER, B. J. F. y RICE, G. E. (1982). *The interaction of reader strategies and the organization of text». Text, 2, 155-192.

MeYer, B. J. F., Young, C. J. y BARTLETT, B. J. (1989). Memory Improved. Reading and Memory Enhancement Across the Life Span Through Strategic Text Structures. New Jersey: Erlbaum.

OAKHILL, J. y GaRnHAM, A. (1988). Becoming a Skilled Reader. Nueva York: Basil Blackwell.

OHLHAUSEN, M. M. y ROLLER, C. M. (1988). \&The operation of text structure and content schemata in isolation in interaction*. Reading Research Quarterly, 23, 1, 70-88.

Owings, R. A., Peterson, G. A., Bransford, J. D., Morris, C. D. y Stein, B. S. (1980). «Spontaneus monitoring and regulation of learning: A comparison of successful and less ssuccessful fifth graders*. Journal of Educational Psychology, 72, 250-256.

PERFETTI, Ch. A. Y ROTH, S. F. (1981). «Some of the interactive processes in reading and their role in reading skill». En A. M. Lesgold y Ch. A. Pefetti (eds.): Interactive processes in reading. Hillsdale, Nueva Jersey: Erlbaum.

Poulson, D., Kintsch, E., Kintsch, W. y Premack, D. (1979). «Children's comprehension and memory for stories*. Journal of Experimental Child Psychology, 28, 379-403.

REDER, L. M. (1985). "Techniques available to Author, Teacher, and reader to improve retention of main ideas of a chapterm. En S. F. Chipman, J. W. Segal y R. Glaser (eds.): Thinking and Learning skills. Vol. 2: Research and Open Questions. Hillsdale, Nueva Jersey: Erlbaum.

ROBINSON, F. P. (1970). Effective study. Nueva York: Harper \& Row.

ROTHKOPT, E. Z. (1976). «W ritting to teach and reading to learn: A perspective on the psychology of written instruction». En N. L. Cage (ed.): The Psychology of Teaching Methods. The seventyfifth yearbook of the National Society for the study of education. Chicago University Press.

RumelHART, D. E. (1975). «Notes on a schema for stories». En D. G. Bobrow y A. Collins (eds.): Representing and Understanding: Studies in Cognitive Science. Nueva York: Academic Press.

- (1977). «Understanding and summarizing brief stories». En D. Laberge y S. J. Samuels (eds.): Basic Processes in reading: Perception and comprehension. Hillsdale. Nueva Jersey: Erlbaum.

- (1980). «Schemata: The building blocks of cognition». En R. J. Spiro, B. C. Bruce y W. F. Brewer (eds.): Theoretical issues in reading comprebension. Hillsdale. Nueva Jersey: Erlbaum.

Rumelhart, D. E., MCClelland, J. L. y grupo PDP (1986). Parallel distributed processing explorations in the microstructure of cognition. Vol. 1. Cambridge, Ma: Bradford Books.

RUMELHART, D. E. y NORMAN, D. A. (1978). *Accretion, tuning, and retructuring: three modes of learningw. En J. W. Cotton y R. L. Klatzky (eds.): Semantic factors in cognition. Hillsdale, N.J.: Erlbaum.

SÁNCHEZ, E. (1988). «Aprender a leer, leer para aprender. Características del escolar con pobre capacidad de comprensión». Infancia y Aprendizaje, 44, 35-57.

- (1990). *Estructuras textuales y procesos de comprensión: un programa para instruir en la comprensión de textos $*$. Estudios de Psicologia, 41, 21-40.

SCARDAmalia, M. y Bereiter, C. (1984). «Development of strategies in text processing». En H. Mandl, N. L. Stein y T. Trabasso (eds.): Learning and comprebension of text. Hillsdale. Nueva Jersey: Erlbaum.

Shallert, D. L., AleXANDER, P. A. y GoeTz, E. (1988). «Implicit instruction of strategies for learning from text.. En C. E. Weinstein, E. T. Goetz y P. A. Alexander (eds.): Leaming and study strategies. San Diego, C.A.: Academic Press.

SCHNOTZ, W. (1984). «Comparative instructional text organization». En H. Mandl, N. L. Stein y T:

STEIN, N. L. y GLENN, C. G. (1979). «An analysis of story comprehensión in elementary school children». En R. O. Freedle (ed.): New Directions in Discourse Processing, Vol. 2. Norwood. Nueva Jersey: Ablex.

TAYLOR, B. M. (1980). «Children's memory for expository text after reading». Reading Research Quarterly, 15, 399-411.

TAYLOR, B. M. y BEACH, R. W. (1984). *The effects of text structure instruction on middle grade students comprehension and production of expository textw. Reading Research Quarterly, 19, 134-146.

TAYLOR, B. M. y SAMUELS, S. J. (1983). «Children's use of text structure in the recall of expository material,. American Educational Research Joumal, 20, 517-528.

THORNDYKE, P. W. (1977). "Cognitive structures in comprehension and memory of narrative discourse». Cognitive Psychology, 9, 77-110.

TURNER, T. N. (1989). «Using textbook questions intelligently». Social Education, 53, 58-60. 
VAN DijK, T. A. (1979). «Relevance assignment in discourse comprehensión». Discourse Processes, 2, 113-126.

- (1980). Macrostructures. Hillsdale, N.J.: Erlbaum.

VAN DIJK, T. A. y KINTSCH, W. (1983). Strategies of discourse comprehension. New York: Academic Press.

VIDAL-ABARCA, E. (1990). «Un programa para la enseñanza de la comprensión de ideas principales de textos expositivos*. Infancia y Aprendizaje, 49, 53-71.

WILHITE, S. C. (1986). Multiple-choice text performance: Effects of heading, questions, motivation and type of retention text question. Ponencia presenta en la American Educational Research Association, San Francisco.

WINNE, P. H. (1982). «Minimizing the black box problem to enhaonce the validity of theories about instructional effects». Instructional Sciences, 11, 13-28.

- (1983). *Training students to process text with adjunt aids». Instructional Sciences, 12, 243-266.

WINOGRAD, P. N. (1983). Strategic difficulties in summarizing texts. Lexington: University of Kentucky.

\section{Extended Summary}

Given the importance of textbooks in education, it is necessary to think about the way we write textbooks. The comprehension strategies of the subjects is one factor must consider in order to improvements as to the quality of instruction. In this sense, this paper provides and assessment of the interactive approach between text and reader features. Within this framework several intervention models designed to facilitate the subject's attainment of reading comprehension are reviewed. The intervention procedures may be based on the written material itself, by means of the writer's own techniques and resources. One of these resources are the signals that direct the attention to the macrostructure they marked.

Another intervention procedures may be based on the subjects characteristics, that is their previous knowledge about the topic of the text or the use and modification of more effective reading strategies on the subject's part. From this latter perspective, training in strategies intended to foster the knowledge and application of the text organizational structure is emphasized. By instructing subjects to employ these strategies they are induced to use a deliberate plan or strategy for remembering what they read. In reading, they are taught to choose the organizational plan used by the writer to organize his or her ideas. In remembering they are taught to use the same organizational plan. 